\title{
The prevalence and clinical profile of adrenocortical deficiency among HIV infected persons in Northern Nigeria
}

\author{
Iorhen E Akase ${ }^{1}$, Abdurazaq G Habib², Adamu G Bakari³, Hamza Muhammad², Ibrahim D Gezawa ${ }^{4}$
}

1. Infectious Disease unit, Lagos University teaching Hospital, Lagos.

2. Infectious Disease unit, Aminu Kano Teaching Hospital, Kano.

3. Endocrinology Unit, Ahmadu Bello University teaching Hospital, Zaria.

4. Endocrinology Unit, Aminu Kano Teaching Hospital, Kano.

\section{Emails:}

A.G. Habib: Email=abdulrazaq_habib@yahoo.co.uk; A.G. Bakari: Email=abgirei@yahoo.com; M. Hamza: Email =drhamza1020@ymail.com; I.D. Gezawa: Email=gezawa01@yahoo.com

\begin{abstract}
Background: Infection with the human immune deficiency virus (HIV) is still a prevalent problem in Africa.

Objectives: The aim of this study was to determine the prevalence of hypocortisolism among patients with HIV and their clinical profile at Aminu Kano Teaching Hospital (AKTH), Kano, Nigeria.

Method: Three hundred and fifty adult patients with HIV infection were recruited from the HIV clinic of AKTH, Kano. Blood samples for serum electrolytes, and cortisol both before and after the short Synacthen test were taken for estimation. Data were analyzed using the SPSS version 20.0 software.

Results: One hundred and eight $(30.9 \%)$ of participants had low baseline serum cortisol levels, while $57(16.3 \%)$ had a low serum cortisol after short synacthen test. There was no significant relationship between the cortisol levels and clinical features of hypocortisolism, WHO clinical stage of HIV, hypernatremia or HAART regimen. There was a negative correlation between the stimulated serum cortisol and duration of diagnosis of HIV, participants BMI and CD4 counts.

Conclusion: The biochemical evidence of hypocortisolism was common among patients infected with HIV, associated with a longer duration of HIV infection. However, none of CD4 counts, clinical features or HAART regimen were associated with hypocortisolism.
\end{abstract}

Keywords: Hypocortisolism, HIV infection, short Synacthen test.

DOI: https://dx.doi.org/10.4314/ahs.v19i2.18

Cite as: Akase IE, Habib AG, Bakari AG, Mubammad H, Gezawa ID. The prevalence and clinical profile of adrenocortical deficiency among HIV infected persons in Northern Nigeria. Afri Health Sci.2019;19(2): 1947-1952. bttps:/ / dx.doi.org/10.4314/abs.v19i2.18

\section{Introduction}

Infection with the human immune deficiency virus (HIV) is still a prevalent problem despite significant strides in its control, with an estimated 36.7 million people infected

\section{Corresponding author:}

Iorhen Ephraim Akase, Infectious Disease Unit, Department of Medicine, Lagos University Teaching Hospital, Idi- Araba, Lagos State, Nigeria. E-mail: akasephraim@yahoo.com Phone: +2348035185940 worldwide, $69.5 \%$ of whom are resident in sub-Saharan Africa. ${ }^{1}$ In Nigeria, about $3.1 \%$ of the population are currently living with HIV infection, amounting to about 3.5 million people. ${ }^{1}$

Adrenal gland dysfunction was one of the earliest endocrine problems documented in people infected with HIV and presenting with AIDS, with several autopsy studies revealing various abnormalities of the adrenal gland. ${ }^{2,3}$ It is thought that the presence of adrenal insufficiency may contribute to increased morbidity in HIV patients, and may serve as a predictor of poor outcomes in patients with HIV infection ${ }^{4}$. Few studies have been conducted in Nigeria to demonstrate the burden of hypocortisolism among HIV patients in Nigeria. A study by Odeniyi et al

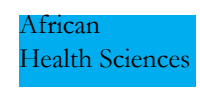

(C) 2019 Akase et al. Licensee African Health Sciences. This is an Open Access article distributed under the terms of the Creative commons Attribution License (https://creativecommons.org/licenses/BY/4.0), which permits unrestricted use, distribution, and reproduction in any medium, provided the original work is properly cited. 
among newly diagnosed HIV patients in Lagos Nigeria showed that $34.8 \%$ of the tested patients had hypocortisolism. ${ }^{5}$ Studies elsewhere in Africa show that hypoadrenalism hypocortisolism is a common problem among patients infected with $\mathrm{HIV}^{6-7}$ and may contribute significantly to both morbidity and mortality among these patients.

Given the significance of hypocortisolism in HIV infection and the paucity of information in Northern Nigeria, the aim of this study was to evaluate adrenal function using the short Synacthen test in this group of patients, and determine the prevalence of hypocortisolism, and the clinical profile of affected patients at the Aminu Kano Teaching Hospital (AKTH), Kano.

\section{Methods}

This study was descriptive cross-sectional study, conducted at the AKTH, Kano. The study subjects included three hundred and fifty (350) consecutive patients with HIV infection presenting at the ART clinic of AKTH Kano. The estimated sample size of 348 was arrived at using the formula: $\mathrm{n}=\mathrm{Z}^{2} \mathrm{pq} / \mathrm{d}^{2.8}$ The prevalence of hypocortisolism in persons with HIV infection was taken to be $34.8 \% .^{5}$ Three hundred and fifty patients (350) were therefore recruited for this study. Recruited patients were adults ( $\geq 18$ years), who were confirmed to have HIV infection according to the serial testing algorithm in accordance with the national guidelines.

Participants were recruited from September 2015 to January 2016. All the recruited patients were interviewed for features suggestive of hypocortisolism. They subsequently underwent laboratory evaluation for the detection of hypocortisolism.

Patients presenting in addisonian crisis, those who were on steroids, and other drugs that could interfere with adrenal function (e.g. ketoconazole, phenytoin, and rifampicin) were excluded from the study.

Patients' clinical data were collected using an interviewer administered questionnaire. Blood samples for serum cortisol estimation were taken at baseline and at $30 \mathrm{~min}$ utes following the administration of $1 \mu \mathrm{g}$ of tetracosactrin (Synacthen). In addition, the patients had blood samples taken at 0 minutes (baseline) for serum urea, electrolytes and creatinine.

After explaining the procedure to the patient, $1 \mu \mathrm{g}$ of Synacthen was prepared by adding $250 \mu \mathrm{g}$ of tetracosactrin
(Synacthen) (1mL) into $499 \mathrm{~mL}$ of normal saline and mixing the solution thoroughly. Each $2 \mathrm{~mL}$ of the diluted solution contained $1 \mu \mathrm{g}$ tetracosactrin (Synacthen). The solution thus prepared was sealed after each usage and stored in a refrigerator at $4-8^{\circ} \mathrm{C}$.

The short Synacthen test was conducted in the morning between 8:00 AM and 9:00AM. All the recruited participants had serum measurement both at baseline and 30 minutes after Synacthen administration. After securing a venous access, the site was observed for the presence of hematoma or bleeding. One $\mathrm{mL}$ of normal saline was then injected to ensure patency of the vein, and $3 \mathrm{~mL}$ of blood was taken at 0 minutes, following which, $1 \mu \mathrm{g}$ of Synacthen was injected intravenously. Another $3 \mathrm{~mL}$ of blood was drawn after 30 minutes. All the blood samples were kept on ice slabs and transported to the laboratory using ice packs for separation and storage of the serum at $-20^{\circ} \mathrm{C}$ until assayed. Estimation of serum cortisol was done after pooling of samples. The Calbiotech ${ }^{\circledR}$ Cortisol ELISA kit (Calbiotech Inc., Spring Valley, CA, USA) was used for the serum cortisol assay. Hypocortisolism was defined as early morning basal serum cortisol less than $145 \mu \mathrm{g} / \mathrm{L}(400 \mathrm{nmol} / \mathrm{L})$ and a 30 minute post-ACTH (stimulated) serum cortisol level of less than $180 \mu \mathrm{g} / \mathrm{L}$ (500 nmol/L).

All data were analyzed using Statistical Package for Social Sciences (SPSS), version 20.0, Chicago, IL USA. Participants' serum cortisol was presented as median with range while other quantitative variables like age, BMI, blood pressure, serum urea and electrolytes were presented as means and standard deviation. The qualitative variables were presented as proportions and percentages. Statistical test of significance at 5\% alpha level was done using the appropriate test statistic, and were considered significant for $\mathrm{p}$ values $<0.05$. The student $\mathrm{t}$-test was used to compare the means of quantitative variables while the Mann-Whitney $\mathrm{U}$ test was used to compare the medians of the stimulated serum cortisol among participants with normal serum cortisol and those with hypocortisolism. The $\chi^{2}$ test was used to compare proportions between two or more groups and to test for association between two nominal scale variables. The linear-by-linear association was used to measure the association between nominal and ordinal variables. Logistic regression analysis was carried out to determine the predictors of hypocortisolism among the 
participants, using clinical features, duration of treatment, BMI, and serum urea and electrolytes as predictors.

The Ethical \& Scientific committee of AKTH approved the study. Written informed Consent was obtained from each participant after demonstrating a satisfactory level of understanding regarding the nature and extent of their involvement and had willingly agreed to participate. Consent on behalf of very sick patients for the study was given by the patients' next of kin after they demonstrated a clear understanding of the nature and extent of the study. Information and data derived were kept confidential and treated as such by the investigators. Participants who were found to have low serum cortisol were referred and linked to the Endocrine clinic of the Aminu Kano Teaching Hospital, Kano.

\section{Results}

The mean (SD) age of the study participants was $39.75 \pm$ 9.22 years; $172(49.1 \%)$ of the participants were male and $178(50.9 \%)$ were females.

\section{Serum cortisol}

Analysis of the baseline serum cortisol showed that 108
(30.9\%) of the 350 study participants had serum cortisol levels below $145 \mu \mathrm{g} / \mathrm{L}$ with a median value of $75.48 \mu \mathrm{g} / \mathrm{L}$ (11.36-144.96). (Median difference $=120.78, \mathrm{p}<0.001$, Mann-Whitney $U$ test). Following the administration of $1 \mu \mathrm{g}$ of ACTH, 57 (16.3\%) participants had measured serum cortisol values of less than $180 \mu \mathrm{g} / \mathrm{L}$ with a median value of $118 \mu \mathrm{g} / \mathrm{L}$ (19.43-179.62). (Median difference = 133.69, $\mathrm{p}<0.001$, Mann-Whitney U test)

\section{Clinical features}

The commonest observed symptom among participants with hypocortisolism was anorexia, which was observed in $13(22.8 \%)$ participants with low stimulated serum cortisol, followed closely by weight loss, fever, lethargy and hyperpigmentation. Abdominal pain occurred in only one participant with low stimulated serum cortisol $(1.75 \%)$. None of the participants with low levels of stimulated serum cortisol reported symptoms of diarrhea. The most commonly documented examination finding among participants with low stimulated serum cortisol was pallor detected in $13(22.8 \%)$ of the respondents. None of the participants with low stimulated serum cortisol levels was noted to have skin hyperpigmentation.

See Table 1.

Table 1 showing some of the symptoms of the participants

\begin{tabular}{llll}
\hline Clinical feature & $\begin{array}{l}\text { Hypoadrenalism } \\
(\mathbf{n = 5 7 )}\end{array}$ & $\begin{array}{l}\text { Normal } \\
(\mathbf{n = 2 7 3 )}\end{array}$ & $\boldsymbol{p}$ value \\
\hline Anorexia & $13(22.8 \%)$ & $57(19.5 \%)$ & 0.56 \\
\hline Weight loss & $10(17.5 \%)$ & $54(18.4 \%)$ & 0.87 \\
\hline Fever & $9(15.8 \%)$ & $44(15 \%)$ & 0.88 \\
\hline Lethargy & $9(15.8 \%)$ & $51(14.6 \%)$ & 0.77 \\
\hline Headache & $6(10.5 \%)$ & $10(3.4 \%)$ & 0.02 \\
Skin rashes & $5(8.7 \%)$ & $29(9.9 \%)$ & 0.79 \\
Abdominal pains & $1(1.75 \%)$ & $9(3 \%)$ & 0.59 \\
\hline Diarhhoea & 0 & $8(2.7 \%)$ & 0.21 \\
Pallor & $13(22.8 \%)$ & $47(16 \%)$ & 0.22 \\
\hline Oral thrush & $2(3.5 \%)$ & $6(2 \%)$ & 0.49 \\
Dyspigmentation & 0 & $4(1.4 \%)$ & 0.38 \\
\hline
\end{tabular}

\section{Duration from diagnosis of HIV}

The highest proportion of the participants with low stimulated serum cortisol was found among those who had been on treatment for 6-10 years (20.4\% [21 out of 103]). The participants that had hypocortisolism were noted to have had a longer duration of HIV compared with those that had normal adrenal function (4.96 years versus 4.38 years, mean difference $=-0.583, \mathrm{p}=0.28)$.

\section{Anthropometry and BP measurement}

Participants with hypocortisolism were noted to have a higher BMI as shown in Table 2. (Mean difference -0.78 , $95 \% \mathrm{CI}=-2.20-0.64, \mathrm{p}=0.28)$.

Postural drop in systolic was observed in 8 of 57 (14.04\%) participants with hypocortisolism compared to 10 of 293 $(3.41 \%)$ with normal function; however, only $7.56 \%$ of the postural drop in systolic drop was dependent on the adrenal status of the participants $(\eta 2=0.00756)$. 
Table 2: Clinical signs and anthropometry of the study participants

\begin{tabular}{|c|c|c|c|c|c|}
\hline Parameter & Category & $\begin{array}{l}\text { Normo- } \\
\text { cortisolismlism }\end{array}$ & $\begin{array}{l}\text { Adreno-cortical } \\
\text { insufficiency }\end{array}$ & $P$ value & $\mathrm{Cl}$ \\
\hline \multirow[t]{5}{*}{ BMI $\left(\mathrm{Kg} / \mathrm{m}^{2}\right)$} & Underweight & 43 & 7 & 0.18 & \\
\hline & Normal weight & 145 & 25 & & \\
\hline & Overweight & 80 & 17 & & \\
\hline & Obese & 22 & 7 & & \\
\hline & Total & 290 & 56 & & \\
\hline \multirow{7}{*}{$\begin{array}{l}\text { Clinical } \\
\text { signs }\end{array}$} & Supine SBP & $127.40(27.34)$ & $120.64(20.74)$ & 0.06 & $1.31-13.4$ \\
\hline & Supine DBP & $85.78(16.25)$ & $81.31(14.81)$ & 0.12 & $0.58-9.24$ \\
\hline & Standing SBP & $122.58(22.43)$ & $119.29(21.58)$ & 0.30 & $3.02-9.59$ \\
\hline & Standing DBP & $83.07(15.58)$ & $81.76(14.97)$ & 0.56 & $-1.31-13.42$ \\
\hline & Pallor & $47(16 \%)$ & $13(22.8 \%)$ & 0.22 & \\
\hline & Oral thrush & $6(2 \%)$ & $2(3.5 \%)$ & 0.49 & \\
\hline & Dyspigmentation & $4(1.4 \%)$ & 0 & 0.38 & \\
\hline
\end{tabular}

\section{Serum electrolytes}

Only $2(3.5 \%)$ of the participants with a low stimulated serum cortisol had elevated serum urea levels., with 12 $(21.1 \%)$ of those with low stimulated serum cortisol noted to have hyponatraemia.

None of the participants with low stimulated serum cortisol had hyperkalaemia, with $12(21 \%)$ of the participants with low stimulated serum cortisol found to have hypokalaemia., as shown in Table 3 below.

A logistic regression analysis was conducted to predict the occurrence of hypocortisolism using duration of treatment, BMI, and serum urea and electrolytes as predictors. A test of the full model against a constant only model was not statistically significant, indicating that the predictors as a set did not reliably distinguish between the presence of hypocortisolism and normal adrenal function $\left(\chi^{2}=17.99, \mathrm{p}=0.59, \mathrm{df}=20\right)$. Nagelkerke's $\mathrm{R}^{2}$ of 0.12 indicated a weak relationship between prediction and grouping. Prediction success overall was $82.2 \%$ (99.1\% for normal adrenal function and $2.1 \%$ for the occurrence of hypocortisolism). The Wald criterion demonstrated that only serum potassium made a significant prediction $(\mathrm{OR}=2.2, \mathrm{p}=0.04)$.

Table 3: Laboratory parameters of participants

\begin{tabular}{llll}
\hline Parameter & \multicolumn{2}{c}{ Frequency (Percentage) } & $P$ value \\
\cline { 2 - 4 } & \multicolumn{1}{c}{ Normal Cortisol } & Hypoadrenalism & \\
Baseline Cortisol $(\mu \mathrm{g} / \mathrm{L})$ & $196.26(151-525)$ & $75.48(11.36-144.96)$ & $<0.001$ \\
Stimulated Cortisol $(\mu \mathrm{g} / \mathrm{L})$ & $251.69(181.91-865.23)$ & $118(19.43-179.62)$ & $<0.001$ \\
Urea $(\mathrm{mmol} / \mathrm{L})$ & $4.23 \pm 1.9$ & $3.90 \pm 1.3$ & 0.21 \\
Sodium $(\mathrm{mmol} / \mathrm{L})$ & $137.53 \pm 6.3$ & $136.81 \pm 5.7$ & 0.42 \\
Potassium $(\mathrm{mmol} / \mathrm{L})$ & $3.84 \pm 0.44$ & $3.87 \pm 0.5$ & 0.64 \\
Chloride $(\mathrm{mmol} / \mathrm{L})$ & $99.77 \pm 6.05$ & $99.81 \pm 5.3$ & 0.78 \\
Bicarbonate $(\mathrm{mmol} / \mathrm{L})$ & $21.68 \pm 2.4$ & $22.37 \pm 2.2$ & 0.04
\end{tabular}




\section{Discussion}

The prevalence of hypocortisolism using the stimulated serum cortisol concentration of $16.3 \%$ as observed in this study was lower than the $34.8 \%$ observed in Lagos by Odeniyi et al 5 although that study comprised only newly diagnosed HAART naïve patients. The prevalence of $30.9 \%$ using basal serum cortisol was similar to that of a study done in South Africa which found a prevalence of $27 \%{ }^{7}$. The South African study however recruited patients who were taking anti-fungals and anti-convulsants at the time of evaluation, both of which are known to interfere with serum cortisol measurements. ${ }^{8,9}$ The prevalence of $16.3 \%$ using stimulated serum cortisol levels is similar to the $19 \%$ found by Meya et $\mathrm{al}^{6}$ in Makerere, although the study was conducted among patients who were admitted with various critical illnesses in HIV (e.g. Tuberculosis - pulmonary and extra-pulmonary, cryptococcal meningitis, kaposi sarcoma, pneumocystis jiroveci pneumonia etc).

Majority of the participants with hypocortisolism in this study as measured by stimulated serum cortisol had no symptoms traditionally thought to be suggestive of hypocortisolism. On the other hand, most of the patients without hypocortisolism had features which may be seen in patients with hypocortisolism. This is consistent with findings by other authors who have noted a poor correlation between clinical features suggestive of hypocortisolism in HIV patients with adrenal insufficiency. ${ }^{5,6,10}$ In the study by Meya et $\mathrm{al}^{6}$, symptoms of fatigue, anorexia, weight loss and vomiting occurred with similar frequency among those who had adrenal insufficiency and those with normal cortisol function.

In fact, most of the studies quoted above found no reliable way of identifying HIV patients based on symptoms, as patients without symptoms were found to have adrenal insufficiency and those with symptoms found to have a normal serum cortisol level at baseline and on stimulation with low dose ACTH. Studies have shown however, that some patients with symptoms of hypocortisolism whose initial serum cortisol tests are normal later develop impaired adrenocortical response on repeat testing with ACTH and overnight Metyrapone test. ${ }^{11,12}$

The significant relationship between serum potassium and hypocortisolism found in our study is in keeping with the established fact that hypocortisolism in HIV negative patients is associated with hyperkalaemia and hyponatrae- mia. ${ }^{13,14}$ In the study by Odeniyi et $\mathrm{al}^{5}$, there was no relationship between serum electrolytes and the occurrence of adrenal insufficiency, a finding reproduced in several studies elsewhere. ${ }^{6,10,11,15}$ Meya et $\mathrm{al}^{6}$ in Makerere also noted that electrolyte levels were normal in subjects with functional adrenal insufficiency and those with normal cortisol function. ${ }^{7}$ In addition, none of hypokalaemia, hyponatraemia or hypoglycaemia was associated with adrenal insufficiency. ${ }^{7}$ In fact, it is generally agreed that the classical findings of hyponatraemia and hyperkalaemia are seldom seen in HIV patients with hypocortisolism. ${ }^{16,17}$ The observed change in the clinical and characteristics among HIV patients with hypocortisolism may be the result of the changing epidemiology of the HIV epidemic. HIV detection is occurring at earlier stages than was obtained earlier in the epidemic. Additionally, routine screening and prophylactic treatment for most ois has led to reduced incidence of destructive and/ or granulomatous lesions of the adrenal gland. It is also worthy of note that patients with functional adrenocortical insufficiency are known to have a residual adrenal reserve, and are therefore less likely to present with the frank features that are seen in patients with symptomatic adrenal insufficiency.

There is much that remains unknown. There is debate as to whether patients with immunosuppression from HIV presenting with hypocortisolism will benefit from steroid replacement at all. Additionally, it is not yet clear the role of persistent inflammation in the setting of HIV and whether this eventually produces adrenal 'exhaustion', with resultant hypocortisolism among patients who have been diagnosed with HIV for long periods.

This study was limited by the cross-sectional design and the consecutive sampling. It would have been ideal to follow-up these participants with hypocortisolism over timeto establish how many eventually developed symptoms of adrenal insufficiency. Additionally, we did not evaluate for ois and possible etiologic entities that were present at the time of study.

\section{Conclusion}

Hypocortisolism detected on biochemical testing is a common problem among patients who are infected with HIV. The occurrence of hypocortisolism among patients infected with HIV cannot be predicted accurately on the basis ofpatients' clinical features or electrolyte abnormalities. Longer duration of HIV infection is associated with hypocortisolism. 
This study further stresses the need for the clinician to have a high index of suspicion when dealing with patients infected with HIV. Ultimately, there is a need to have algorithms that specify which patients infected with HIV will get screened for adrenal function as the features of hypocortisolism are non-specific and may not be recognized in HIV because they are masked by the features of other opportunistic infections.

\section{Acknowledgement}

We wish to thank the management of Ahmadu Bello University for the logistic and laboratory support in the course of this study.

\section{Conflict of interest}

The authors declare that there is no conflict of interest regarding the publication of this paper.

\section{Acknowledgement}

We wish to thank the management of Ahmadu Bello University for the logistic and laboratory support in the course of this study.

\section{References}

1. Global report: UNAIDS report on the global AIDS epidemic 2015

2. Guarda LA, Luna MA, Smith Jr. JL, Mansell PW, Gyorkey F, Roca AN. Acquired immune deficiency syndrome: postmortem findings. American Journal of clinical pathology. 1984 May; 81(5): 549-557.

3. Tapper ML, Rotterdam HZ, Lerner CW, Al'Khafaji K, Sietzman PA. Adrenal necrosis in the acquired immunodeficiency syndrome. Ann Intern Med. 1984; 100:239-241. Pubmed

4. Lo J, Grinspoon SK. Adrenal function in HIV infection. Current opinion in Endocrinology, Diabetes and Obesity. 2010; 17:205-9.

5. Odeniyi IA, Fasanmade OA, Ajala MO, Ohwovoriole AE. CD4 count as a predictor of adrenocortical insufficiency in persons with human immunodeficiency virus infection: How useful? Indian Journal of Endocrinology and Metabolism. 2013; 17:1012-1017.
6. Meya DB, Katabira E, Otim M, Ronald A, Colebunders $\mathrm{R}$, Denise N, et al. Functional Adrenal Insufficiency among critically ill patients with Human Immunodeficiency Virus in a resource limited setting. African Health Sciences. 2007; 7(2): 101-107.

7. Ekpebegh CO, Ogbera AO, Longo-Mbenza B, Blanco-Blanco E, Awotedu A, Oluboyo P. Basal cortisol levels and correlates of hypoadrenalism in patients with $\mathrm{Hu}-$ man Immunodeficiency Virus Infection. Med Princ Pract. 2011; 20:525-9. Pubmed

8. Todd TT. The effects of HIV-1 infection on endocrine organs. Best Practice \& Research Clinical Endocrinology \& Metabolism. 2011; 25: 403- 413. Pubmed

9. Danoff A. Endocrinologic complications of HIV infection. Medical Clinics of North America. 1996; 80(6): 1453-1469.

10. Abbott M, Khoo SH, Hammers MR, Wilkins EGL. Prevalence of cortisol deficiency in late HIV disease. Journal of Infection. 1995; 31: 1-4.

11. Marik PE, Kiminyo K, Zaloga GP. Adrenal insufficiency in critically ill patients with HIV. Critical Care Medicine. 2002; 30: 1267-1273.

12. Eledrisi MS, Verghese AC. Adrenal insufficiency in HIV infection: A review and recommendations. American Journal of Medical Sciences. 2001; 321: 137-144.

13. Charmandari E, Nicolaides NC, Chrousos GP. Adrenal insufficiency. The Lancet. 2014; doi.org/10.1016/ S0140-6736 (13)61684-0.

14. Steven Soule. Addison's disease in Africa—a teaching hospital experience. Clinical Endocrinilogy. 1999; 50: 115120. Pubmed

15. Raffi F, Brisseau JM, Planchon B, Remi JP, Barrier $\mathrm{JH}$, Grolleau JY. Endocrine function in $98 \mathrm{HIV}$ infected patients: a prospective study. AIDS. 1991: 5; 729-733. Pubmed

16. Zapanti E, Terzidis K, Chrousos G. Dysfunction of the Hypothalamic-Pituitary-Adrenal axis in HIV infection and disease. Hormones, 2008; 7: 205-216.

17. Post FA, Soule SG, Willcox PA, Levitt NS. The spectrum of endocrine dysfunction in active pulmonary tuberculosis. Clinical Endocrinilogy. 1994;40:367-71. 